21st Particles and Nuclei International Conference (PANIC 2017)

International Journal of Modern Physics: Conference Series

Vol. 46 (2018) 1860082 (6 pages)

(C) The Author(s)

DOI: $10.1142 / \mathrm{S} 2010194518600820$

\title{
The Belle II Experiment: Status and Physics Prospects
}

\author{
Jake Bennett \\ Physics Department, Carnegie Mellon University, 5000 Forbes Ave \\ Pittsburgh, Pennsylvania, 15213, USA \\ jvbennett@cmu.edu
}

Published 3 May 2018

\begin{abstract}
The Belle II experiment is now under construction at the KEK laboratory in Japan. This project represents a substantial upgrade to both the Belle detector and the KEKB accelerator. The Belle II experiment will record 50/ab of data, a factor of 50 more than that recorded by Belle. This large data set, combined with the low backgrounds and high trigger efficiencies characteristic of an $e^{+} e^{-}$experiment, should provide unprecedented sensitivity to new physics signatures in $B$ and $D$ meson decays, and in $\tau$ lepton decays. The experiment is scheduled to begin physics running in 2018 .
\end{abstract}

\section{Introduction}

The Belle II experiment inherits an extremely successful physics program from the first generation $B$-factories, Belle at KEKB and BaBar at SLAC, which recorded a combined sample of over $1.5 \mathrm{ab}^{-1}$, corresponding to about 1.25 billion $B \bar{B}$ decays. Among the many notable discoveries and measurements is the experimental confirmation of the CKM mechanism as the source of CP violation (CPV) in the standard model (SM) with the first measurements of the CKM parameter $\sin (2 \beta)$ in $2001^{1,2}$. Subsequent measurements of the CKM parameters by the $B$-factories and other experiments have, thus far, been in excellent agreement with the $\mathrm{SM}^{3}$. Nevertheless, a number of important questions remain unanswered. These include whether there are alternative sources of $\mathrm{CPV}$ and the possibility for new particles or interactions. The Belle II experiment will carry on the legacy of the first generation $B$-factories to seek answers to these questions.

The Belle II experiment is the first major new experiment in the LHC-era. In contrast to LHC experiments operating at the energy frontier, wherein new particles are sought via direct production that is limited by the accessible beam energy,

This is an Open Access article published by World Scientific Publishing Company. It is distributed under the terms of the Creative Commons Attribution 4.0 (CC-BY) License. Further distribution of this work is permitted, provided the original work is properly cited. 


\section{J. Bennett}

Belle II will search for NP at the intensity frontier. With the unprecedented luminosity accessible at the upgraded SuperKEKB facility, Belle II will seek indirect evidence of NP by searching for signatures of new particles or processes through measurements of suppressed flavor physics reactions or deviations from SM predictions. Any observed discrepancies could then be interpreted in terms of NP models. This enables a probe for NP to energies above $10 \mathrm{TeV}$.

\section{The SuperKEKB Accelerator}

In order to support the Belle II physics program at the intensity frontier, significantly more data than that collected at the first generation $B$-factories is needed. The ultimate goal of Belle II is to collect $50 \mathrm{ab}^{-1}$ of data. This will be possible due to the major upgrade of the KEKB accelerator facility, in Tsukuba, Japan ${ }^{4}$. To achieve a much higher luminosity, a number of accelerator components have been replaced. This includes a new positron source and damping ring, new beam pipes and bellows, and sophisticated new final-focus quadrupole magnets near the interaction point (IP), as well as the upgraded Belle II detector. With these modifications, the design luminosity of the new SuperKEKB accelerator is $8 \times 10^{35} \mathrm{~cm}^{-2} \mathrm{~s}^{-1}, 40$ times greater than that of KEKB.

In order to achieve this design luminosity, SuperKEKB will make use of the nanobeam scheme ${ }^{5}$, in which the luminosity is greatly increased by reducing the beam size to the order of a few hundred atomic layers. Other modifications include a reduction in the beam asymmetry and therefore a reduction of the center-of-mass (CM) boost by nearly a factor of two relative to that at KEKB. This improves the hermiticity of the detector, but makes measurements of time-dependent CPV (TDCPV) more challenging. However, the improved vertexing capabilities of the upgraded Belle II detector should compensate for this effect.

\section{The Belle II Detector}

The Belle II detector contains a number of new or upgraded subsystems. A new vertex detector (VXD) lies immediately outside the beryllium beam pipe and consists of two layers of silicon DEPFET pixels surrounded by four layers of double-sided silicon strips. Charged particle tracking is achieved with a large-radius, small-celled central drift chamber (CDC) filled with helium-ethane gas $\left(50 \% / 50 \%\right.$ He: $\left.\mathrm{C}_{2} \mathrm{H}_{6}\right)$. Surrounding the drift chamber is a sophisticated new particle identification (PID) detector consisting of two subsystems. The barrel region of the detector contains the imaging time-of-propagation detector, which consists of long quartz bars and a system of spherical mirrors that focus the cherenkov photons onto finely segmented multi-channel-plate phototubes and provides timing resolution better than 100 ps. The PID subsystem in the forward endcap region consists of a proximity focusing ring-imaging cherenkov counter that uses silicon aerogel with a varying index of refraction. Outside of the PID detectors is an electromagnetic calorimeter (EMC), 


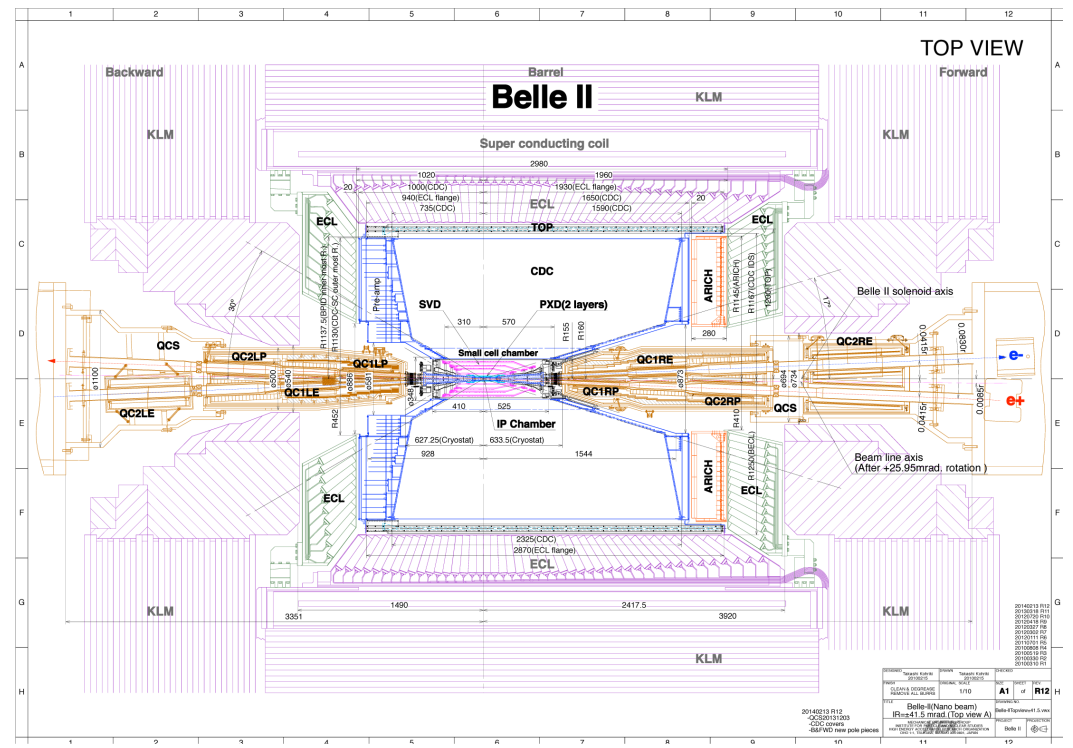

Fig. 1. A schematic, top view of the Belle II detector ${ }^{6}$.

consisting of $\mathrm{CsI}(\mathrm{Tl})$ crystals. In order to cope with the much higher beam backgrounds expected at SuperKEKB, the EMC electronics have been upgraded to use waveform sampling. A superconducting solenoid magnet provides a uniform $1.5 \mathrm{~T}$ magnetic field within the tracking region. The iron yoke of the magnet is instrumented with scintillating fiber detectors and glass resistive place chambers, which act as a $K_{L}$ and muon detector (KLM). A schematic view of the Belle II detector is shown in Fig. 3. With the significant upgrades of the detector subsystems, Belle II is expected to perform similar to or better than Belle, despite the expected twenty-fold increase in beam backgrounds.

In addition to the detector components, the readout of the detector and the offline computing have also been upgraded. The data acquisition system (DAQ) must accommodate a maximum level one trigger rate of $30 \mathrm{kHz}$, which is $100 \%$ efficient for hadronic events. The recorded output is expected to include about 30 GB per second. Offline computing at Belle II will be achieved using distributed computing on the grid following the LHC model. This is necessary to manage the processing of the massive Belle II data sets as well as the production of large montecarlo (MC) samples and to support the many concurrent user analysis jobs expected at Belle II. A number of successful campaigns to produce large MC samples have been executed with the Belle II distributed computing system since 2013.

\section{Advantages of SuperKEKB and Belle II}

There are a number of advantages that can be leveraged for the physics program at SuperKEKB and Belle II. Running at $\Upsilon(4 \mathrm{~S})$ threshold, SuperKEKB produces a 
very clean sample of quantum correlated $B^{0} \bar{B}^{0}$ pairs that can be used in TDCPV measurements as well as for other $B$ physics studies. Belle II will also have a much higher flavor-tagging efficiency than at LHCb and can measure both $K_{s}$ and $K_{L}$, which impacts most TDCPV measurements. Dalitz plot analyses and missing mass analyses are also relatively straightforward at a $B$-factory. Belle II also has the distinct advantage of the efficient reconstruction of neutral particles like $\pi^{0}$ and $\eta$ mesons. Additionally, a large sample of tau lepton decays will enable measurements of rare decays and searches for lepton flavor violation (LFV). Notably, given the differences between the detectors, the systematic uncertainties for measurements by Belle II will be quite different than those of LHCb. Any sign of NP seen by one experiment should be confirmed by the other.

One of the most powerful advantages of a physics program at a $B$-factory is the ability to fully reconstruct one of the $B$ mesons produced in $\Upsilon(4 \mathrm{~S})$ decays to tag the flavor of the other $B$ meson, determine its momentum, and isolate its decay products. This "full reconstruction tagging" is an excellent tool for missing energy and missing mass analyses such as the precise measurement of the CKM parameter $\mathrm{V}_{u b}$ in $B$ meson decays to $\mathrm{X} l \nu$ and searches for NP in $B$ meson decays to $\tau \nu$ or $K \nu \nu$.

\section{Belle II Physics Program}

PANIC 2017 featured a number of contributions describing different aspects of the Belle II physics program, including quarkonium physics, CPV sensitivity, and CKM metrology, among others. A full review of the Belle II physics program will be published in the report from the Belle II Theory Interface Platform (B2TiP) ${ }^{6}$. This report will also include a full description of the detector, software, and analysis tools. A few highlights from the physics program are given here.

Some of the physics studies that could be possible with early data include quarkonium spectroscopy. There has been considerable progress recently in Lattice $\mathrm{QCD}^{7}$ and Belle II has the opportunity to search for some of the missing states in the bottomonium spectrum. There is also much to be done to quantify and confirm the presence of the plethora of XYZ states that have been observed recently.

Another possible source of early physics from Belle II include light dark matter searches. For example, ISR production of a dark photon, which then decays to invisible particles. Such studies require a non-trivial, dedicated trigger. With the good calorimetry, KLM efficiency, and hermeticity of the detector, Belle II should have improved sensitivity to channels like this, with only $20 \mathrm{fb}^{-1}$, relative to other experiments ${ }^{6}$. Projected upper limits are shown in Fig. 5.

With the much larger data samples anticipated at Belle II, studies of the flavor anomaly in $R(D)$ and $R\left(D^{*}\right)$ will be accessible. As of September 2017, the combined significance of the disagreement between the SM prediction and measurements of these ratios by $\mathrm{LHCb}$ and the $B$-factories is about $4.1 \sigma^{8}$. Belle II should be able to confirm the excess with about $5 \mathrm{ab}^{-1}$ of data. 

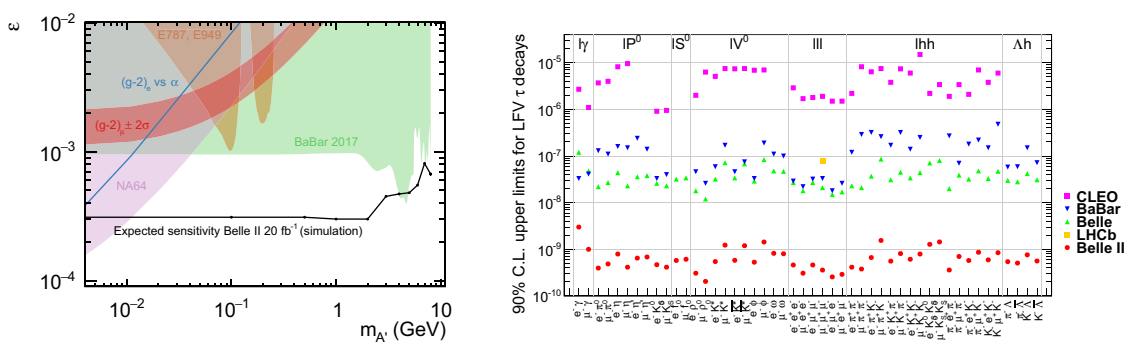

Fig. 2. (Left) Projected upper limits on the strength $\epsilon$ of the kinematic mixing of a dark photon to the CM photon for the process $e^{+} e^{-} \rightarrow \gamma A^{\prime}$, with $A^{\prime} \rightarrow$ invisible with a $20 \mathrm{fb}^{-1}$ data sample at Belle II (solid black curve) ${ }^{6}$. (Right) Upper limits for LFV $\tau$ decays with the full $50 \mathrm{ab}^{-1}$ data sample at Belle $\mathrm{II}^{6}$.

One of the most important questions that Belle II hopes to investigate is whether there are any new $\mathrm{CP}$ violating phases. Most theories involving NP include additional CP-violating phases. Some of these allow for large deviations from SM predictions for $B$ meson decays. Belle II will search for new sources of CPV by comparing mixing-induced $\mathrm{CP}$ asymmetries in penguin transitions with tree-dominated modes. For example, TDCPV measurements in $b$ to $s$ transitions such as $B$ decays to $\phi K^{0}$, $\eta^{\prime} K^{0}$, and $K^{0} K^{0} K^{0}$ can be compared with results from $B$ decays to $J / \psi K^{0}$. Any discrepancies in the two measurements would provide unambiguous evidence for NP.

In addition to being a $B$-factory, Belle II will also be a $\tau$ factory, producing large samples of $\tau$ decays that can be used to search for LFV, which is highly suppressed in the SM and therefore provides a clean probe for NP effects. Hadron machines are not competitive in $\tau$ decay measurements due to trigger and track $p_{T}$ limitations, making $\tau$ decays uniquely studied at $B$-factories. With the full $50 \mathrm{ab}^{-1}$ sample, Belle II can access LFV decay rates more than an order of magnitude smaller than Belle.

\section{SuperKEKB/Belle II Schedule}

Commissioning of the SuperKEKB accelerator began in early 2016. The "BEAST" phase 1 detector, a simple background commissioning detector consisting of components like diodes, TPCs and crystals, was used to perform single beam background studies. The goals of phase 1 commissioning included the tuning of accelerator optics, vacuum scrubbing, beam studies, and validation of the Belle II beam background simulations. A full report on the results of the phase 1 commissioning is to be submitted to NIM-A ${ }^{9}$.

In the time following phase 1 , a number of Belle II detector components have been installed, including the barrel PID system in May of 2016, the CDC in October 2016 and the endcap PID detector in August 2017. The final-focus quadrupole magnets were also installed and tested in late 2016 and early 2017. The Belle II detector was than "rolled-in" to the IP on April 11, 2017. Global cosmics running 


\section{J. Bennett}

with the final 1.5T solenoid field took place in July and August 2017 and included readout integration of all of the installed sub-detectors and the central DAQ.

Phase 2 commissioning will take place in late 2017 and early 2018 and will include commissioning of the damping ring and main ring of the SuperKEKB accelerator. This will lead into phase 2 data taking in early 2018, which will include the first operation of the accelerator with the final-focus magnets and first collisions. While the VXD will not be installed, the "BEAST-VXD" detector, consisting of the beam background commissioning detector along with one octant of the VXD, will be used along with the outer Belle II detector. The goals of phase 2 include verification of the nanobeam scheme, continued beam background studies, especially in the VXD volume, and first physics studies without vertexing.

Later in 2018, the vertex detectors will be installed, following phase 2 commissioning. These sophisticated new detectors should provide significant improvement in vertex resolution with respect to that at Belle, even with the higher beam backgrounds that are expected at SuperKEKB. Once the VXD is installed, phase 3 running will begin with the full Belle II detector, working toward the goal of collecting $50 \mathrm{ab}^{-1}$ of data over the next decade.

\section{Summary}

The SuperKEKB accelerator and Belle II detector represent major upgrades of the KEKB accelerator and Belle detector, ushering in the era of the next generation, super- $B$-factory. Many accelerator and detector components have been upgraded and replaced. The Belle II software and analysis tools have also been greatly improved. These efforts will make possible the rich Belle II physics program, which is both complementary and competitive with existing experiments and the energy frontier program. With successful phase 1 operation in 2016 and cosmic data taking with integrated subsystems and the central DAQ in 2017, the Belle II collaboration is moving into phase 2 commissioning and first physics in 2018. Full detector operation will begin with phase 3 running in late 2018 or early 2019 .

\section{References}

1. B. Aubert et al., Phys. Rev. Lett. 87, 091801 (2001).

2. K. Abe et al., Phys. Rev. Lett. 87, 091802 (2001).

3. CKMFitter Group (J. Charles et al.), Phys. Rev. D 91, 073007 (2015).

4. Y. Ohnishi et al. Prog. Theor. Exp. Phys. 2013, 03A001 (2013).

5. M. Baszczyk et al. INFN-13-01-PI, LAL-13-01, SLAC-R-1003, arXiv:1306.5655 (2013).

6. E. Kou, P. Urquijo, The Belle II Physics Book, to appear in Prog. Theor. Exp. Phys..

7. S. Godfrey and K. Moats, Phys. Rev. D 92, 054034 (2015).

8. S. Hirose et al. Phys. Rev. Lett. 118, 211801 (2017).

9. A. Aloisio et al., First Measurements of Beam Backgrounds at SuperKEKB, to appear in Nucl. Instrum. Meth. A. 